JOSÉ RAÚL ZAVALA

\title{
DESCENTRALIZACIÓN, PROGRAMAS FOCALIZADOS Y CORPORATIVISMO POLÍTICO EN MUNICIPIOS DEL CHACO
}

\author{
1994/1995[1]
}

\begin{abstract}
El autor es Master en Vivienda y Urbanismo (A.A. London Arq.), Becario Externo del CONICET (Argentina), Profesor
\end{abstract} Adjunto de la Facultad de Arquitectura (UNNE, Argentina).

\begin{abstract}
RESUMEN. Este artículo presenta las consecuencias ocultas de la distribución desigual del gasto social durante el proceso de descentralización en el marco de los programas de ajuste estructural en la Argentina. El autor basó su investigación en datos elaborados a partir de una encuesta exploratoria realizada en ocho municipalidades de la provincia del Chaco. Los resultados muestran como los programas focalizados son distribuidos de acuerdo a pertenencia política. Los programas son administrados y distribuidos desde el nivel federal directamente a los municipios sin tener participación agencias del nivel provincial. En este contexto los municipios compiten entre ellos con el objetivo de obtener los recursos del nivel nacional. El artículo culmina presentando y discutiendo algunas estrategias e ideas para mejorar las relaciones entre municipios. La intención es ayudar a conformar una estrategia común que permita el fortalecer del nivel local para poder afrontar el sistema desigual de distribución de los gastos sociales del gobierno federal.
\end{abstract}

ABSTRACT. DECENTRALIZATIO, FOCALISED PROGRAMMES AND POLITICAL COORPORATIVISM IN CHACO MUNICIPALITIES 1994-1995.

This article describes the hidden consequences of uneven social expenditure distribution, driven by decentralization process within the structural adjustment programmed in Argentina.

The author bases this research on data collected from an exploratory survey done in eight municipalities of the Chaco province. These findings show how political bias marks the path of focalized programs. These programs are allocated from national level by passing provincial agencies control and divert to the municipal level. In doing so, the municipalities compete between each other in order to gain resources from the national level. This article ends with a discussion on strategies and ideas to enhance the relationship between municipalities. The aim is to convert a common strategy toward a local empowerment in order to face the uneven social distribution of the federal government expenditure.

\section{INTRODUCCIÓN}

El presente trabajo está centrado en la observación de la relación de ocho Municipios del Chaco y el Estado Nacional. Este último en el rol de gestor y administrador de fondos para el financiamiento del desarrollo municipal, relegando al Municipio la promoción, fomento, orientación y protagonismo de su autodesarrollo.

Los elementos observados fueron los cambios en los procedimientos de la gestión pública y en los modos de financiar el desarrollo de pequeños municipios en el marco de las políticas nacionales de ajuste estructural.

Las proposiciones que guían este trabajo son las siguientes:

n El proceso iniciado con la Reforma del Estado en la República Argentina (1989) en lo referente al mejoramiento de las condiciones de vida en los municipios presenta una variada oferta de programas que no existían, ni en su cantidad, ni en calidad, en el modelo anterior. 
n Estos nuevos recursos no son aprovechados plenamente por los destinatarios finales (municipios y sus comunidades), por que: a)existe un grado de desarrollo institucional en el sector público y privado que no se ajusta a las condiciones que se establecen desde el Estado Nacional; b) la obligación de constituir garantías o contrapartes financieras limitan el acceso a los programas. Si a ello se suman los desacuerdos en el interior de los Concejos Municipales para avalar o no endeudamientos del municipios se presenta como una seria traba al ingreso de inversiones.

n Los criterios implícitos en la distribución de los recursos por parte del Estado Nacional a los municipios siguen la tendencia de potenciar la reproducción política del partido gobernante, acentuando las desigualdades entre municipios de un mismo departamento.

Las conclusiones del trabajo conducen a:

n Analizar las principales constricciones que debilitan la capacidad de gestión de los gobiernos locales que en distintas instancias y momentos de negociación con el gobierno federal puedan mejorar las condiciones generales, en términos de: a) optimizar las condiciones de acceso, b) propender a una distribución equitativa y democrática de los recursos sociales.

n Ponderar las potencialidades locales como generadoras de desarrollo, incorporando su propia visión y peculiaridades como contraparte al tecnocratismo de los organismos federales.

Este trabajo explora las condiciones dinámicas y constrictivas que tienen los municipios para acceder a planes, programas y financiamientos para sus proyectos de desarrollo (cuando existe o es inducido externamente).

\section{ASPECTOS METODOLÓGICOS}

\section{Dinámica y temporalidad}

Distinguimos dos procesos con escalas temporales distintas, uno opera como contexto del otro: el primero involucra los cambios en la reproducción del Estado Argentino iniciado en 1989, a través de las reformas administrativas del aparato estatal, la búsqueda de equilibrios fiscales, privatización de empresas estatales, descentralización de funciones y apertura del mercado local. El segundo proceso está referido a las modificaciones que siguieron a nivel provincial y en el nivel municipal, en 1994 y 1995 respectivamente, para adaptar sus cuerpos normativos a las establecidas por el gobierno Federal.

Ambos procesos han tenido dinámicas -tensiones y desarrollos- diferenciados. Mientras que el primero tiene un horizonte de medio y largo plazo para observar los resultados de la estrategia adoptada, en el segundo, los efectos son casi inmediatos, como hemos podido determinar a partir de esta investigación.

Las dinámicas descriptas someramente arriba, nos permite diferenciar dos temporalidades. Este trabajo estará acotado a estudiar los cambios en los niveles locales de la estructura administrativa, que hemos señalado como segundo proceso, cuyo inicio lo podemos definir en función de dos hechos relevantes: Ias modificaciones a la Constitución de la Provincia del Chaco en 1994, y las adecuaciones de las Leyes Orgánica Municipales en 1995.

La razón de esta opción reside en que, habida cuenta que existe gran cantidad de investigaciones de los proceses de reforma del estado y sus implicancias territoriales a nivel macro, esta investigación pretende aportar elementos para la discusión desde una mirada local, o desde una sub-región afectada por los fenómenos globales.

Por lo tanto tenemos procesos de cambios significativos en las relaciones estado-sociedad, y entre organismos del mismo estado a distintos niveles. 
El estudio de estos procesos se desarrolla desde dos perspectivas analíticas complementarias: un análisis diacrónico -descripción de la sucesión de los acontecimientos-, y un análisis sincrónico del estado de situación en el momento de realización de la investigación. Para esto último realizamos una encuesta en ocho municipios de segunda y tercera categoría de la provincia del Chaco, que aportó el elemento necesario para confirmar o descartar tendencias en el proceso.

Se pretende, por lo tanto, realizar un primer trabajo en una escala acotada, que permita comprobar o descartar las hipótesis, como así también los instrumentos, y en una segunda fase realizar un trabajo de mayor alcance.

\section{Encuadre teórico y preguntas preliminares}

La investigación abordó:

n Los cambios institucionales en la relación Municipio-Estado Provincial. y,

n Las desigualdades de la relación entre Municipios y el Estado Nacional, el tema central de este artículo.

La hipótesis de partida es que una nueva forma de territorialidad política esta emergiendo a la luz de este incipiente esquema distributivo producto de la reforma estructural del estado. Al no contar con políticas regionales que dirijan ciertos y determinados recursos hacia las distintas regiones que tiene el país la distribución de la ayuda para el desarrollo esta sesgada por arbitrariedades por parte del Estado Nacional.

Las preguntas que este trabajo dilucidará son las siguientes:

n ¿Cómo afectan, las políticas de ajuste estructural al conjunto de municipios de segunda y tercera categoría?

n ¿Que condiciones de incorporación deberían de poseer estos para ser sujetos de las incipientes políticas de descentralización?

Por otra parte, la lógica economicista y pro-mercado que imperaba desde el Ministerio de Economía de la Nación, y que por entonces parecía fuera de toda discusión, planteaban que el país tenía "territorios no viables"[2] con los cuales había que tomar drásticas medidas. La idea de que el país tiene regiones "ganadoras" y "perdedoras" derivaría tarde o temprano en políticas "correctivas" que acentuarían las diferencias, siguiendo esa lógica eficientísta.

Precisamente los municipios analizados en la muestra, formaban parte de estos "territorios no viables" de allí que nos interrogamos:

n ¿Que factores contribuían para que estos municipios y sus fuerzas productivas se "empeñaran" en seguir desarrollando actividades en esos "inviables" lugares?

n ¿ Bajo qué racionalidad el estado debería seguir "soportando los gastos" generados?

n ¿Cómo eran las modalidades de inversión del desarrollo en municipios de segunda y tercera categoría en donde el peso de las poblaciones era bajo, y no constituían atractivos para la inversión del capital privado en términos de producción de bienes y servicios?

n ¿Cómo, y bajo qué condiciones operarían estos municipios en el Ajuste Estructural?

\section{Encuesta: la muestra y las variables medidas}

Las primeras exploraciones que realizamos nos indicaron que no podríamos contar con información de fuentes secundarias. 
El organismo encargado de manejar las relaciones con los municipios a nivel provincial carecía de información referidas a cuales eran las vinculaciones de los municipios con el nivel Nacional. A nivel Nacional cinco ministerios estaban encargados de dirigir sus programas -un total de 127- hacia los 1.922 municipios que tiene el país, por lo tanto la dispersión de la información era de tal magnitud que no se correlacionaban con los medios que disponíamos. No esta demás ponderar la importancia de esta información no disponible al momento que realizamos nuestra investigación, que debería aportar significativos datos sobre el universo de los municipios de la provincia

La información primaria fue laboriosamente confeccionada con la colaboración del personal municipal, secretarios e intendentes que encontraron la tarea muy útil para sus propias instituciones. No está de más aclarar que la filigrana tejida en la tarea de la encuesta se debió a que los municipios encuestados carecen de una sección de estadística o de seguimiento de gestión o en otras palabras de un ámbito de control de la gestión municipal. Por lo tanto, cada encuesta demandó entre uno y tres días en confeccionarse, a ese ritmo nos hubiera llevado siete meses en cubrir los sesenta y ocho municipios de la provincia con los medios que disponíamos.

Finalmente tomamos la decisión de dejar a un lado en esta investigación los municipios de primera categoría[3] y tomar para la muestra municipios de segunda y tercera ya que tendrían mas dificultades para financiar su desarrollo, en el marco de la eficacia administrativa que demandan las políticas de Ajuste Estructural.[4] Y nos permitiría observar mejor las contradicciones de los procesos definidos antes.

Otro factor que alimenta esta opción es la carencia de estudios sobre municipios pequeños que dieran referencias respecto de su situación bajo las condiciones y modalidades impuestas por la reforma del Estado.

La encuesta se llevó a cabo en ocho municipios: "Las Palmas", "La Leonesa" e "Isla del Cerrito" del Departamento de Bermejo; "Laguna Blanca", "Colonia Popular", del Departamento Libertad; "Fontana" del Departamento San Fernando; "Margarita Belén", "Colonia Benítez" del Departamento de $1^{\circ}$ de Mayo, todos lindantes con la capital de la Provincia: Resistencia. Este número representa el 12\% del total de municipios de segunda y tercera categoría, con partidos a cargo del ejecutivo municipal con representación parlamentaria a nivel nacional.

La encuesta contiene las siguientes variables; "composición de ingresos corrientes y no corrientes", "composición de las erogaciones corrientes, y no corrientes", "tipo y extensión de servicios municipales", "tipo y calidad de vinculaciones con organismos descentralizados de servicios", "programas, convenios con organismos nacionales".

Buscábamos, en este trabajo de campo exploratorio, hallar evidencias que dieran veracidad a las hipótesis que expresaban las formas en que se sustenta el desarrollo de los municipios bajo las políticas de ajuste estructural.

\section{ESTADO, DESCENTRALIZACIÓN E IDEAS}

Unas de las consecuencias perseguidas por la ley de Reforma del Estado bajo las políticas de ajuste estructural en la República Argentina, sería la generación de una mayor participación de los municipios a partir de las políticas de descentralización, siguiendo las imposiciones de los organismos internacionales de crédito que reconocen su inicio a nivel mundial hace quince años atrás aprovechando la circunstancia de las crisis de los Estados Benefactores y la necesidad de su rediseño. De hecho más de 75 países con poblaciones entre 5 a 12 millones de habitantes, en 1990 habían comenzado a transferir algunas formas de poder desde el Estado Nacional a los municipios (Ramirez. 1997: 2).

En el caso Argentino el "Programa de Saneamiento Financiero y Desarrollo Económico de las Provincias Argentinas" (PROVINCIAS I), fue el medio de convencimiento utilizado por el Estado Nacional y los organismos internacionales de financiamiento (Banco Mundial, Banco Interamericano de Desarrollo) a comienzos de los 90' para que las provincias y los municipios aceleraran sus reformas fiscales y mejoraran la eficiencia del gasto en concordancia con el ajuste a nivel Nacional. 
El programa fue concebido como "premio" a las provincias y municipios que realizaban transformaciones fiscales en concordancia con lineamientos federales. El financiamiento estuvo dirigido al desarrollo institucional e inversiones en infraestructura, estas últimas primaron sobre la primera por su alta repercusión política. Los subproyectos fueron diseñados, implementados, supervisados y ejecutados por las provincias. El financiamiento alcanzó a US\$ 400 millones, las contrapartidas provinciales alcanzó a US\$ 175 millones. Un segundo tramo fue gestionado por la Nación que se denominó PROVINCIAS II, cuyo costo total alcanzo a US\$ 321 millones, (Zapata. 1998: 268-269).

Estos programas sirvieron como puentes para el desarrollo de otros créditos no sólo de las mencionadas instituciones financieras sino del Eximbank de Japón operando directamente con las provincias (Zapata, op. cit). En otras palabras se produjo una extensión del mercado del crédito sobre una demanda cautiva. La descentralización en el país siguió una estrategia de trasladar los gastos a los niveles de gobierno que tengan condiciones económicas-financieras para absorber créditos del mercado financiero internacional, en otras palabras, reducir el endeudamiento del gobierno central y propender a incrementar el provincial y o municipal (Cuadro 1).

Los argumentos generales de descentralización del estado han seguido por lo menos estas dos visiones:

n La descentralización es una política a seguir cuando hay que definir los niveles de gobierno para efectuar el gasto y recaudar los recursos, considerando que la población beneficiada por un bien público está determinada por las característica espaciales del bien, y que no todos los bienes públicos tienen el mismo alcance geográfico (Zapata, 1998:264).

n La descentralización limita y refuerza a las autoridades locales como así también potencia la incorporación de las organizaciones comunitarias que en conjunción facilitarían una mayor democratización de la sociedad en su conjunto ya que propenderían a reducir desigualdades sociales y económicas allí donde los requerimientos son más apremiantes.

Cuadro 1. Argentina: Gastos por Sector y Nivel de Gobierno, 1980-84 / 1994

(Secretaría de Programación Económica "El Federalismo en Argentina" por J. Sanguintti y M. Tettamanti, 1995. Cit. en Zapata 1998).

\begin{tabular}{|c|c|c|c|c|c|c|c|}
\hline Sector & $\begin{array}{l}\text { Gob. Central } \\
\text { 1980-84 }\end{array}$ & Gob. Central & $\begin{array}{l}\text { Gob. Provincial } \\
\text { 1980-84 }\end{array}$ & $\begin{array}{l}\text { Gob. Provincial } \\
1994\end{array}$ & $\begin{array}{l}\text { Gob. } \\
\text { Municipal } \\
\text { 1980-84 }\end{array}$ & $\begin{array}{l}\text { Gob. } \\
\text { Municipal } \\
1994\end{array}$ & Total \\
\hline Educación básica & 33,7 & 0,4 & 63,7 & 96,5 & 2,6 & 3,2 & 100 \\
\hline Educación superior & 83,9 & 78 & 16,1 & 22 & 0 & 0 & 100 \\
\hline Salud básica & 14,8 & 12,7 & 75,3 & 73,7 & 10 & 13,6 & 100 \\
\hline Saneamiento & 32,4 & 7,9 & 67,6 & 92,1 & 0 & 0 & 100 \\
\hline Vivienda & 13,5 & 6,5 & 75,4 & 69,6 & 11,1 & 23,9 & 100 \\
\hline Bienestar social & 65,9 & 16,2 & 34,1 & 83,8 & 0 & 0 & 100 \\
\hline Servicios urbanos & 0 & 0 & 3,6 & 6,7 & 96,4 & 93,3 & 100 \\
\hline Total & 70,7 & 54,2 & 25 & 38,5 & 4,3 & 7,4 & 100 \\
\hline
\end{tabular}

La descentralización como política en la Argentina, ha seguido el primer criterio. El provincias I y II operó como un caso testigo para los municipios y provincias "indisciplinadas". A pesar del aparente criterio técnico con el cual operaron estos, la discrecionalidad política fue sin duda el criterio para la distribución de los recursos, como este trabajo desarrollará mas adelante.

Ramírez (1997: 4) llama la atención frente al conflicto que aparece cuando se incorpora la dimensión política que estos discursos carecen. En el caso de Argentina, esta variable ha generado un importante retraso en la definición de la descentralización del estado. Nuestro argumento es que el principal obstáculo para su concreción radica en la visión tecnocrática de la descentralización por una lado, y por otro la modalidad corporativa de la reproducción política, que propicia el fortalecimiento del poder del funcionario político antes que el fortalecimiento del ciudadano. 
Esta modalidad corporativa de reproducción política, tiene dos polos funcionales, por un lado el Estado nacional con la administración de los subsidios y créditos de variadas modalidades de acceso y por otro los 1.922 municipios que componen el país.

Si realizamos una analogía con el mercado donde los Municipios son la demanda y el Estado Nacional la oferta. Se establece una relación asimétrica en términos de poder. Donde la 'oferta' es dispensada en consonancia a 'acuerdos tácticos' entre este nivel y el provincial. Por lo tanto la 'demanda' esta condicionada a otros factores que exceden su ámbito local. Acentuando las necesidades y debilidades locales que se traduce en debilitar la gestión institucional ya que se hace evidente que esta no tiene fuerzas suficientes para presionar sobre la "calidad y forma distributiva de la oferta".

Por lo tanto están condicionadas en su capacidad de inversión para el desarrollo por las presentes condiciones que establece esta modalidad corporativa, que, por obvias cuestiones de reproducción política del gobierno, éste retrasará las políticas para el traspaso real de recursos a los municipios. En la Argentina se ha justificado esta situación, desde el punto de "vista administrativo" poniendo todas las virtudes del lado del Estado Nacional y las incapacidades del lado de los municipios.

Coraggio (1991:187) han formulado que: "El eje administrativo juega como gran justificador del económico: se privilegia el nivel local del Estado (Municipios, Provincia) y se apoya su capacidad de administrar y gestionar servicios; se propugnan fórmulas de justificación de proyectos de inversión donde la recuperación y el subsidio son la norma..."

Los modelos tecnocráticos de descentralización que ha hegemonizado el discurso del estado nacional ligado a estrategias macroeconómicas. Esta concepción, que fue presentada como eficiencia estatal, no ha sido otra fase depurada de "clientelismo tecnocrático". Donde la dimensión política no es ni siquiera mencionada o es intencionalmente dejada de lado. Nuestro trabajo se dirige entonces, a reconstruir una empiria que de cuenta de las condiciones económicas y como estas son reforzadas por las políticas distributivas que distorsionan aún mas las precarias condiciones de los municipios, aún los agraciados en la distribución desigual.

\section{ANTECEDENTES}

\section{Datos de población}

La provincia del Chaco con sus 918.711[5] habitantes se ubica en el noveno lugar en la tabla de habitantes, en relación con las restantes 23 provincias que componen la República Argentina.

Su base productiva es primaria por excelencia, aportando el $75 \%$ de la producción algodonera del país.

Está ubicada en el nordeste del país y limita con las provincias de: Formosa al norte, Santiago del Estero al oeste, con Santa Fe al Sur y finalmente al este con los Ríos Paraná y Paraguay, ríos limítrofes con la República del Paraguay y la provincia de Corrientes (Argentina).

En sus $99.633 \mathrm{~km} 2$, contiene 25 departamentos compuestos por 68 municipios divididos en tres categorías según el número de población.

Como nos muestra el Cuadro 2, la provincia sigue el patrón de alta urbanización, con el 58\% de su población concentrada en ocho municipios. De los cuales el $30 \%$ se ubican en su capital provincial, Resistencia.

Cuadro 2. Municipios de la Provincia del Chaco presentados por Categorías, Frecuencia, Población en $\%$ del total Provincial y Superficie en Km2. (Elaboración propia en base a "Los Municipios Actuales, sus categorías, superficie, población" publicado en "El Municipio" órgano de difusión de la Subsecretaria de Asuntos Municipales, Ministerios de Gobierno, Justicia y Educación, Gobierno de la Provincia del Chaco, 1995).

\begin{tabular}{llll}
\hline Categoría & Frecuencia & Población & Superficie \\
\hline $1^{0}$ & 8 & $58,3 \%$ & $12,35 \%$
\end{tabular}




\begin{tabular}{llll}
$2^{\circ}$ & 24 & $26,45 \%$ & $40,9 \%$ \\
$3^{\circ}$ & 36 & $15,16 \%$ & $46,72 \%$ \\
TOTAL & 68 & $100 \%$ & $100 \%$ \\
\hline
\end{tabular}

La baja densidad de población es la característica de los municipios de $2^{\circ}$ y $3^{\circ}$ categoría. Mientras que los municipios de $2^{\circ}$ categoría contienen el $26,45 \%$ de la población distribuidos en 24 municipios. La diferencia se incrementa en los municipios de $3^{\circ}$ categoría donde el $15,16 \%$ de la población se distribuye en 36 de ellos.

Por lo que se puede establecer con claridad la alta diferenciación que existen entre categorías de municipios a partir de los datos que nos proporciona la densidad de población (Cuadro 3).

Cuadro 3. Municipios de la Provincia del Chaco presentados por Categoría, Frecuencia, Densidad en Km2. (En base a Sinopsis Estadística de Argentina, Dirección de Difusión del Instituto de Estadísticas y Censo de la República Argentina, 1997)

\begin{tabular}{lll}
\hline Categoría & Frecuencia & Densidad $(\mathrm{Km} 2)$ \\
\hline $1^{\circ}$ & 8 & $40,3 \%$ \\
$2^{\circ}$ & 24 & $5,5 \%$ \\
$3^{\circ}$ & 36 & $2,7 \%$ \\
\hline
\end{tabular}

\section{Aportes desde la dispersión de población}

Podemos reconocer dos patrones de agrupación de población a las cuales denominaremos de "relativa concentración" y de "marcada dispersión". El primer patrón ha sido objeto de atención de programas de investigación a nivel Latinoamericano y Europeo sobre el proceso de rápida urbanización de las "ciudades intermedias"[6] en América Latina en los 90' y que renueva el interés sobre el tema en esta primera década de este milenio.[7] Si bien este enfoque ha señalado las profundas y rápidas modificaciones del medio urbano, con la aparición de nuevos actores y nuevas formas de gestión de la ciudad. También es destacable la carencia de investigaciones sobre lo que aquí denominamos áreas de "marcada dispersión", donde las condiciones de producción y de reproducción social presentan contriciones particulares que este trabajo desarrolla y cuyas consecuencias inmediatas pueden ser apreciadas en los fenómenos de las ciudades intermedias.

\section{RESEÑA DE LOS CAMBIOS EN LA ESTRUCTURA J URIDICA}

A continuación, se presenta una breve referencia de las modificaciones producidas en la Constitución Provincial y en particular en la Ley Orgánica Municipal que dieron la adecuación institucional a la provincia para hacerse "benefactora" de las nuevas condiciones de "crecimiento".

La constitución de la provincia del Chaco fue promulgada en el año 1957, -por las paradojas de la historia- no tuvo un sesgo liberal decimonónico como la Constitución Nacional, al cual otras provincias replicaron. En este caso, ésta, incorporó mecanismos de democracia directa; estableció que el Estado provincial es el motor de desarrollo de la economía junto al sector privado; por lo tanto estableció un sistema financiero y la distribución de tierras públicas para la producción primaria. Definió que los servicios públicos fueran estatales con cogestión de los usuarios y también estableció una destacada autonomía municipal.

Durante 1994, el partido gobernante en el Chaco[8] lanza una convocatoria para reformar la constitución provincial, en particular el articulo 54, que establece como bien social los servicios públicos y abre las puertas para la privatización del Banco Provincial. Para ello fue necesario un llamado a plebiscito vinculante. Realizado éste, la población se manifestó contraria a tales modificaciones. En lo concerniente a los municipios se ampliaron las facultades de estos para adecuarse a la futura descentralización.

Un año después se promulga la Ley Orgánica Municipal № 4233, que su artículo $3^{\circ}$ establece con claridad lo qué se entiende por autonomía municipal: «...significa instaurar un Gobierno Municipal 
dotado esencialmente de la facultad de disponer de sus bienes y recursos, del cumplimiento de sus fines propios y de la organización y administración de los servicios locales; conformando un régimen autónomo de carácter técnico-administrativo y funcional que convierte a los municipios en factores de la descentralización territorial.»

De esta manera queda establecida la autonomía. Pero, anteriormente a las reformas, este aspecto es un punto de conflicto entre las municipalidades y el gobierno provincial, ya que los municipios tenían limitado el uso de los fondos coparticipables que les asignaba la provincia.

La adecuación institucional de la Provincia del Chaco a las políticas de Ajuste estructural tuvo como resultado final:

n No se avanza en aspectos de privatización de servicios públicos,[9] en la escala que pretende el Gobierno Federal, sí, en el reforzamiento de mecanismo de democracia directa;

n A nivel municipal se alcanza la autonomía necesaria para poder gestionar su desarrollo con miras a lo que por entonces aparece como eminente, las políticas de descentralización,[10] que darían una nueva dinámica a los municipios dado que estos no estarían condicionados a la única fuente financiera, las partidas Provinciales distribuidas hasta entonces.

\section{DESPLIEGUE DEL AJ USTE ESTRUCTURAL EN SU ETAPA COMPENSATORIA}

Las políticas de descentralización municipal operaron en la Argentina más como factor de imposición de los organismos internacionales de crédito, que por aceptación y buena voluntad política del estado nacional (PROVINCIAS I y II explicadas anteriormente).

Cuando los organismos internacionales lanzan las políticas compensatorias para reducir las consecuencia del incremento de la pobreza producto del ajuste estructural, de nuevo el gobierno muestra gran eficiencia en la generación de programas sociales, que en realidad eran programas cerrados al cual se le agrego una denominación local.

Como otra fase de un mismo proceso que comenzó con los programas PROVINCIAS I y II, el gasto social se focaliza y se materializa con varios programas de créditos y subsidios entre 1994 y 1995 por parte de la Nación, a los municipios de todo el país.

Si el PROVINCIAS I y II fueron los elementos compensatorio para las provincias que privatizaron sus bancos y sus empresas de servicios públicos, la batería de programas sociales sería la herramienta compensatoria para los municipios del país. Con esta política una nueva territorialidad se desplegaba.

Sobre el discurso de la eficiencia del gasto público el estado nacional supervisa los programas que son «descentralizados» a los municipios de todo el país. Eran tan descentralizados que se hacía complicado gestionar los 127 programas que había en agosto de 1995, fecha en que se realiza la encuesta. Esta complicación pareciera que fue exprofesamente desplegada para facilitar el espacio de "operadores" que hacían la articulación entre un conjunto de municipios de una determinada provincia con el ministerio encargado de otorgar estos programas descentralizados.

El elemento sorpresa fue sin duda la gran variedad de programas dirigidos a distintas «población objeto». El estado erradicaba las políticas universales y replicaba los modelos del Banco Mundial de las políticas focalizadas, que no fue más que el reforzamiento de la segregación social.

En sus inicio esta nueva estrategia estaba dirigida a conformar una nueva base política del gobierno que no fue sencillo decodificar por entonces. En este contexto, las provincias quedaban afuera del circuito de programas que coadyuvaba a la descentralización municipal, pero estos continuaban con la inercia donde los presupuestos les eran «bajados» desde la provincia, siendo en realidad administradores locales del dinero provincial. Con este nuevo escenario los municipios tuvieron que hacer lo opuesto a lo que su práctica habitual cual es salir a buscar conexiones políticas con los "operadores" que les permitiera llegar a los programas. 


\section{Municipios en la 'era' de la descentralización, el lado osc uro de la autonomía.}

Buscando la eficiencia del gasto desde el estado nacional, las condiciones de acceso a inversiones ligadas al desarrollo económico de los municipios, o sea la inversión que en el mediano plazo se traduciría en un incremento por tazas y servicios, la creación de nuevas fuentes de trabajo genuinas estaban ligadas a la capacidad de endeudamiento del municipio.

En otras palabras, las inversiones en bienes de capital y fijos demandaban contrapartidas municipales para ser otorgadas. El escenario por lo pronto se redujo notablemente ya que el nivel de endeudamiento de los municipios era en general nulo. Esto obligó a conducir la búsqueda a tomar programas que no demandasen tales compromisos.

En el pasado las inversiones en infraestructura y equipamientos estaban contenidas en las previsiones presupuestarias de un ministerio, sea este provincial o nacional. Los municipios jugaban un rol pasivo en ese esquema o a lo sumo influían para "traer obras al pueblo".

Ahora las inversiones están dirigidas directamente a los municipios (ya no mediatizan los organismos ramales). Los ministerios del nivel nacional, con sus variadas ofertas de programas operan como entes financiadores. Por lo tanto, todas las inversiones deben tener contrapartidas o bien ser garantizadas por ingresos corrientes municipales.

\section{RESULTADOS}

\section{Municipios descentralizados y capacidad de endeudamiento}

El punto de interés está, entonces, en analizar cuál es la capacidad económica de los municipios, ya que a partir de las nuevas condiciones descriptas, éstos cobran una ponderación determinante para la toma de nuevos ingresos.

Más adelante se retoma el análisis de las ofertas de programas del estado nacional. En los párrafos que siguen nos concentramos en analizar las economías de los municipios encuestados.

El Cuadro 4, presenta las fuentes de ingresos de los presupuestos municipales. El promedio general de un $77 \%$ esta originado en los ingresos por coparticipación provincial. Estos recursos son originados en el nivel de la coparticipación Federal. Por lo tanto la disponibilidad de los ingresos corrientes de los municipios están expuestos a la cadena de "manipulaciones financieras"[11] por parte de la provincia y de la Nación.

Cuadro 4. Composición de los ingresos en los presupuestos municipales, en \% y promedios. (

Porcentajes basados sobre los presupuestos proyectados para 1995).

\begin{tabular}{llll}
\hline & Categoría & $\begin{array}{l}\text { Ingresos en \% por } \\
\text { Coparticipación }\end{array}$ & $\begin{array}{l}\text { Ingresos } \\
\%\end{array}$ \\
\hline Fontana & $2^{\circ}$ & 65 & 35 \\
La Leonesa & $2^{\circ}$ & 63 & 38 \\
Las Palmas & $2^{\circ}$ & 67,07 & 32,93 \\
Laguna Blanca & $3^{\circ}$ & 72 & 28 \\
Isla Del Cerrito & $3^{\circ}$ & 100 & - \\
Margarita & $3^{\circ}$ & 71,9 & 28,07 \\
Belén & & & 10 \\
Colonia & $3^{\circ}$ & 90 & 10 \\
Benítez & & & \\
Colonia & $3^{\circ}$ & 90 & $23 \%$ \\
Popular & & $77 \%$ & \\
\hline PROMEDIOS & & & \\
\hline
\end{tabular}


Cuadro 5. Municipios según otorgamiento de Aportes del Tesoro Nacional. (Fuente Elaboración Propia).

\begin{tabular}{lll}
\hline & $\begin{array}{l}\text { Aportes del Tesoro } \\
\text { Nacional Otorgados }\end{array}$ & $\begin{array}{l}\text { Signo Partidario del } \\
\text { Gobierno Municipal }\end{array}$ \\
\hline Fontana & Sí & PJ \\
La Leonesa & NO & UCR \\
Las Palmas & S/D & PJ \\
Laguna Blanca & NO & ACHA \\
Isla Del Cerrito & Sí & PJ \\
Margarita Belén & Sí & PJ \\
Colonia Benítez & NO & ACHA \\
Colonia Popular & Sí & PJ \\
\hline
\end{tabular}

Ref.: PJ: Partido Justicialista; UCR: Unión Cívica Radical; ACHA: Acción Chaqueña.

Los ingresos propios promedian un 23\% y están compuestos entre otros, por tasas aplicadas a la producción primaria y los servicios conexos. El inconveniente que presenta su recaudación es la fluctuación que este rubro tiene, ya que las tazas se aplican siguiendo los ciclos productivos de la actividad primaria, base económica de los ocho municipios de nuestra encuesta. Esta se encuentra condicionada a los fenómenos meteorológicos, combinación de ciclos lluvias-sequías, que colocan en situación de volatilidad a este vital recurso. Por lo tanto los ingresos corrientes por tazas son por lo general impredecible al momento de establecer los presupuestos anuales.

Complementa el panorama de ingresos no corrientes de los municipios, una fuente que opera selectivamente y que son los aportes no reintegrables del Tesoro Nacional a municipios con graves problemas financieros. Como nos muestra el cuadro siguiente solamente tienen problemas financieros los municipios cuyo signo partidario es "similar" al que "lo distribuye" a nivel Nacional, mientras que en los municipios cuyos partido gobernante son de la "oposición no existen zozobras financieras".

A manera de síntesis puede afirmarse que las principales fuentes de ingresos que componen los presupuestos municipales están fuertemente influenciados por factores externos, tales como:

n Relación Gobierno Provincial y Nación en el envío de las partidas a la Provincia de la coparticipación federal.

n Condiciones meteorológicas desfavorables que reducen la capacidad de recaudación a los municipios y...

n La discriminación-favoritismo en el otorgamiento de ingresos de carácter extraordinario como los Aportes del Tesoro Nacional.

Otra cara de la moneda de los presupuestos municipales da la composición de los egresos (Cuadro 6). El principal sentido que tiene analizarlos, radica en poder "predecir" que variaciones deberían producir en la dirección de estos para que los municipios puedan incorporarse el nuevo modelo de gestión de desarrollo.

Cuadro 6. Composición de los Egresos en \%. (Fuente Elaboración Propia).

\begin{tabular}{lllllll}
\hline & Categoría & $\begin{array}{l}\text { Sueldos } \\
\text { Personal }\end{array}$ & $\begin{array}{l}\text { Bienes de } \\
\text { Consumo y } \\
\text { Servicios }\end{array}$ & Subsidios & Deudas( ${ }^{*}$ ) & $\begin{array}{l}\text { Obras } \\
\text { Públicas }\end{array}$ \\
\hline Fontana & $2^{\circ}$ & 90 & 3 & 1 & - & 6 \\
La Leonesa & $2^{\circ}$ & 51 & 6 & 1 & 29 & 13 \\
Las Palmas & $2^{\circ}$ & 59 & 10 & 1 & 25 & 5
\end{tabular}




\begin{tabular}{lllllll} 
Laguna Blanca & $3^{\circ}$ & 58 & 14,5 & 4,5 & - & 23 \\
Isla Del Cerrito & $3^{\circ}$ & 90 & 3 & 2 & - & 5 \\
Margarita Belén & $3^{\circ}$ & 46 & 20 & 4 & 22 & 8 \\
Colonia Benítez & $3^{\circ}$ & 87 & 10 & - & - & 3 \\
Colonia Popular & $3^{\circ}$ & 80 & 4 & 1 & 7 & 8 \\
\hline PROMEDIOS & $70 \%$ & $9 \%$ & $2 \%$ & $10 \%$ & $9 \%$ \\
\hline
\end{tabular}

${ }^{\star}{ }^{*}$ Los casilleros vacíos corresponden a moratoria de pago a terceros.

Partiendo del supuesto que ninguna nueva fuente de ingreso se incorporare en el mediano plazo, los municipios tendrán que "rediseñar" sus gastos para optimizar su capacidad de endeudamiento a la luz de las nuevas reglas de juego impuestas desde el nivel Nacional, y que los municipios han aceptado en cierta medida cuando realizaron los adecuamientos institucionales.

Los municipios aportan puestos de trabajo estables y transitorios, en cantidad y calidad que han permitido a muchas comunidades mantener aceptables niveles de subsistencia. Esta dimensión no es considerada dentro de la lógica eficientista de los ajustes que la nación exige a los municipios. Queremos remarcar que el principal desacierto de las políticas de reducción de gasto se centra en la reducción de personal sin ponderar alternativas que los incorpore en otros procesos productivos. Consecuentemente, estas facilitan el proceso de incrementar nuevas formas de pobreza en el corto plazo.

Por citar algunos ejemplos, en instituciones provinciales el personal mas calificado tomara «retiros anticipados» quedando el organismo seriamente debilitado en su capacidad funcional. En otros, en cambio, significó la salida de personal poco calificado quedando el organismo con estructuras gerenciales pero sin estructuras operativas. En los dos casos fueron atraídos hacia el «cuenta propismo» que en un par de años los capitales invertidos fueron consumidos. Generándose nuevas formas de pobreza.

En el contexto de las precarias condiciones económicas que tienen los municipios analizados, llevar adelante esta concepción tecnocrática tiene consecuencias a distintos niveles. Si analizamos que el $70 \%$ de los egresos está destinado al rubro personal, que no guarda relación con la columna de Bienes de Consumo y Servicios como así también el de Mantenimiento de Obras Públicas, donde los porcentuales son extremadamente bajos. Una interpretación es que los municipios están mas comprometidos a mantener personal sobrecargando la administración y reduciendo al mínimo la prestación de servicios. Si aceptamos esto como válido podría aceptarse también que las demandas de reestructuración que exige el estado nacional estaría muy lejos de alcanzarse, por la gran distancia existente entre el esquema o los escenarios posibles a los que se quiere arribar desde la administración central y las condiciones reales en la cual se encuentran los municipios que analizamos.

Otra visión nos ofrece la columna de deudas, donde el 50\% de los municipios encuestados estaban en moratoria de pagos a acreedores, esta situación reduce sus capacidades funcionales a corto plazo.

Intentando establecer conclusiones respecto de la composición de egresos de los municipios encuestados, puede afirmarse que:

n Los municipios presentan una escasa capacidad de endeudamiento como así también de inversión, dado que el conjunto suma un $19 \%$. Sólo un $9 \%$ esta disponible para ser dirigido al mantenimiento y construcción de pequeñas obras de infraestructura. Como resultado de esto, habría graves limitaciones al momento de decidir nuevas deudas e inversiones (como demandan los programas nacionales de desarrollo), en el plazo inmediato.

n La consecuencia mas inmediata en la reducción de personal sería el corte de la cadena de pago a nivel doméstico, como emergente más notorio, que pondría en juego a corto plazo la legitimidad social de los municipios, ya que históricamente ha sido un factor determinante[12] en el uso de fuerza de trabajo y en la preservación de la paz social. De hecho a nivel municipal no conocemos casos de reducciones de personal.[13] Otro mecanismo economicistas sugerido por el Estado nacional fue la formula de traspasar personal estatal al sector privado. 
Con este panorama de arrastre que presentan los municipios, se aprestan a incorporarse a los nuevos mecanismos económicos-financiero de gestión del desarrollo. En el tópico siguiente se desarrolla, básicamente la relación municipios-Estado nacional en lo referente al uso de la oferta de programas por parte de los municipios.

\section{COMO SE DISTRIBUYEN LOS RECURSOS NACIONALES A LOS MUNICIPIOS ANALIZADOS}

El análisis de la distribución desigual no se presenta claramente en un solo acto, por lo tanto iremos desplegando distintos grados de acercamientos a los datos que conducen hacia nuestro objetivo, el cual es demostrar la desigualdad en la distribución de los recursos públicos.

Nuestra proposición operativa es: La reforma de Estado con sus variadas ofertas de programas para el desarrollo de los municipios ha mantenido formas distributivas desiguales ya que los criterios seguidos priorizan la discriminación política (corporativismo político) al momento del otorgamiento, estableciendo inversiones desiguales entre municipios de un mismo departamento.

El análisis desarrollará los siguientes criterios; a) presentaremos los programas en términos de cantidad y estado de los mismos, b) los objetivos perseguidos y; c) como fueron distribuidos.

En el Cuadro 7 los programas fueron medidos por municipios en cantidad y estado de realización, (ejecutados, en ejecución, en gestión). El número de programas distribuidos en los distintos municipios, revela una relativa equidad. Salvo el caso de Isla del Cerrito, que es un territorio turístico a escala regional y con intenciones de convertirse en atractivo internacional, que hace que a él concurran inversiones para la industria turística que no tienen los demás municipios. Los programas presentan una igualitaria distribución y anticipan a la luz de los datos de gestión, una tendencia a aumentar su número.

Cuadro 7. Municipios y Programas, Ejecutados, Otorgados y en Gestión. (Fuente Elaboración Propia).

\begin{tabular}{llllll}
\hline & $\begin{array}{l}\text { Programas } \\
\text { Ejecutados }\end{array}$ & $\begin{array}{l}\text { Programas } \\
\text { En } \\
\text { Ejecución }\end{array}$ & $\begin{array}{l}\text { Programas } \\
\text { En Gestión }\end{array}$ & $\begin{array}{l}\text { Total } \\
\text { Programas }\end{array}$ & $\begin{array}{l}\text { Signo } \\
\text { Partidario }\end{array}$ \\
\hline Fontana & 2 & 4 & 2 & 8 & $\mathrm{PJ}$ \\
La Leonesa & 2 & 2 & 3 & 7 & UCR \\
Las Palmas & 2 & 2 & 3 & 7 & $\mathrm{PJ}$ \\
Laguna Blanca & 2 & 2 & 1 & 5 & $\mathrm{ACHA}$ \\
Isla Del Cerrito & 2 & 2 & 9 & 15 & $\mathrm{PJ}$ \\
Margarita Belén & 3 & 3 & 1 & 7 & $\mathrm{PJ}$ \\
Colonia Benítez & 2 & 1 & 1 & 4 & $\mathrm{ACHA}$ \\
Colonia Popular & 2 & 3 & 1 & 6 & $\mathrm{PJ}$ \\
\hline PROMEDIOS & & 2.1 & 2.3 & 2.6 & 7,3 \\
\hline
\end{tabular}

Ref.: PJ: Partido Justicialista; UCR: Unión Cívica Radical; ACHA: Acción Chaqueña.

Otro acercamiento al conjunto de la problemática de la distribución nos ofrece el Cuadro 8 donde se presentan los municipios según los objetivos que cubren los programas. El rubro de la construcción es el más favorecido, básicamente por el inmediato impacto económico, por la creación de puestos de trabajo y el efecto en el consumo cotidiano. El segundo en orden, es el programa de empleos que administran los municipios. Estas dos líneas de inversión están dirigidas al sector activo, mientras que al sector pasivo asiste el programa alimentario que le siguen en orden de prelación.

La característica en común que tienen los programas contenidos en el cuadro ultimo, (incluido el de Regulación de Títulos de Propiedad), es que son subsidios directos. A los municipios solo les cabe 
completar los formularios disponibles y las proximidades de las fechas eleccionarias determinará los tiempos en la entrega de los subsidios.[14]

Cuadro 8. Municipios agrupados por Departamentos, según Subsidios recibidos, Signo Partidarios y Distribución de programas según objetivos. (Fuente Elaboración Propia).

\begin{tabular}{|c|c|c|c|c|c|c|c|}
\hline & ATN & $\begin{array}{l}\text { Signo } \\
\text { Partidario }\end{array}$ & A & $\mathrm{B}$ & $\mathrm{C}$ & $\mathrm{D}$ & $\mathrm{E}$ \\
\hline \multicolumn{8}{|l|}{ BERMEJO } \\
\hline La Leonesa & NO & UCR & 2 & - & - & 1 & 1 \\
\hline Las Palmas & SD & PJ & 2 & - & - & 1 & 1 \\
\hline Isla Del Cerrito & sí & $\mathrm{PJ}$ & 4 & - & - & - & - \\
\hline \multicolumn{8}{|l|}{ S. FERNANDO } \\
\hline Fontana & sí & $\mathrm{PJ}$ & 1 & - & 1 & 2 & 2 \\
\hline \multicolumn{8}{|l|}{ LIBERTAD } \\
\hline Colonia Popular & sí & PJ & 2 & - & 1 & 2 & - \\
\hline Laguna Blanca & NO & $\mathrm{ACHA}$ & 2 & - & 1 & 1 & - \\
\hline \multicolumn{8}{|l|}{$1^{\circ} \mathrm{DE}$ MAYO } \\
\hline Margarita Belén & sí & $\mathrm{PJ}$ & 3 & 1 & 1 & 1 & - \\
\hline Colonia Benítez & NO & $\mathrm{ACHA}$ & 2 & - & 1 & - & - \\
\hline TOTALES & & & 18 & 1 & 5 & 8 & 4 \\
\hline
\end{tabular}

Ref.: ATN: Aportes del Tesoro Nacional subsidios; PJ: Partido Justicialista; UCR: Unión Cívica Radical; ACHA: Acción Chaqueña; A: Obras de Infraestructuras, Equipamiento Social, Obras especiales (refacción, restauración, equipamiento turístico); B: Programas de Desarrollo de Producción Primaria; C: Programas Alimentarios; D: Programas de Empleos; E: Programas de Normalización de solares, donaciones.

Un párrafo particular merece el programa de dinamización productiva, del municipio de Margarita Belén. Diríamos que este es el "único de la especie" que obedece a los criterios «eficientista». Es un crédito dirigido a la optimización de la producción de un conjunto de agricultores que se asumen como deudores, para ello la municipalidad acuerda ser el garante, por lo tanto los Concejos Municipales deben disponer de una porción de la coparticipación municipal como garantía de la operación.

De allí que estas condiciones de acceso al crédito sea una barrera para la extensión del mismo en el ámbito rural. No es una paradoja que habiendo necesidad objetiva de recursos para la producción primaria, este no sea aprovechado por los agricultores. Algunas respuestas posibles pueden esgrimirse desde los factores socioculturales si analizamos las experiencias pasadas.

En los inicios del los '70, con las organizaciones de base de los agricultores, denominadas Ligas Agrarias (Rozé, 1992), que permitió la defensa de los precios de la producción primaria, frente a los industriales y al estado Nacional, culminó con una represión a mediado de los '70 a los agricultores que pugnaban por cobrar los precios acordados antes de la siembra y que los acopiadores se negaban a aceptar.

Mas tarde al inicio de los '80 la Dictadura Militar lanza un programa de financiamiento a agricultores, con el objeto de optimizar la producción con miras al mercado internacional. Mientras éstos, se endeudaban en moneda estadounidense, el precio del algodón a nivel de mercado internacional se encontraba muy alto, por lo tanto las expectativas eran muy positivas. Las condiciones del mercado internacional cambian y en el transcurso del primer y segundo año los precios se devalúan de manera tal que las deudas contraídas no pueden ser absorbidas por los beneficios de la producción y se genera una bancarrota importante del sector.

Haciendo una prospectiva diríamos, que esta vía de financiamiento deberá superar varios momentos de "confiabilidad" y que será extendido al conjunto de programas del Estado Nacional antes de que exista una aceptación del conjunto social al cual está dirigido. 
Básicamente por ser programas originados en la tecnocracia ministerial -replicados de las recetas de los organismos de crédito internacional, alejados de las condiciones de realización del desarrollo que tienen los municipios, y particularmente -como habíamos adelantado- sin instancias de participación por parte de estos y sus comunidades, hace que el subsidio siga siendo la única modalidad a la cual pueden acceder estas poblaciones para el mejoramiento de sus condiciones físico ambientales.

Tampoco se han facilitado situaciones para desarrollar mecanismos de consultas intermunicipales que pudieran sintetizar necesidades y prioridades que se articulen en políticas a ser consensuadas con los niveles nacionales.

Pero para que esto sea posible deberían eliminarse ciertas coerciones propias del corporativismo político, que utiliza recursos del estado para su reproducción política, creando desigualdades entre ciudadanos y territorios.

En el Cuadro 9 presentamos el número de programas distribuidos según partido político a cargo del gobierno municipal. De los ocho municipios encuestados 5 de ellos pertenecen al Partido Justicialista (PJ), dos a Acción Chaqueña (ACHA) y solo uno a la Unión Cívica Radical (UCR). Debemos aclarar que esta es una representación del universo de la provincia, de allí el mayor peso que tiene los municipios del PJ en la muestra. Pero midiendo el conjunto de municipios por promedio de programas otorgados encontramos una preeminencia en cantidad a favor del PJ, comparando con las dos restantes fuerzas políticas.

Cuadro 9. Distribución de Programas Ejecutados y en Ejecución según Partidos Políticos a cargo del Gobierno Municipal. (Fuente Elaboración Propia).

\begin{tabular}{lll}
\hline Partido Político & $\begin{array}{l}\text { Promedios } \\
\text { Programas } \\
\text { por Municipios }\end{array}$ & $\begin{array}{l}\text { de } \\
\text { Ejeculados } \\
\text { + En Ejecución }\end{array}$ \\
\hline $\begin{array}{l}\text { Unión Cívica } \\
\text { Radical }\end{array}$ & 4 & 4 \\
$\begin{array}{l}\text { Acción Chaqueña } \\
\begin{array}{l}\text { Partido } \\
\text { Justicialista }\end{array}\end{array}$ & 3,5 & 7 \\
\hline
\end{tabular}

Mientras que el Cuadro 10 presenta que la potencialidad de acceso a programas está definitivamente volcado al partido oficial $(\mathrm{PJ})$ en seis veces más que los otros. No sin razón, se podría plantear, que estas diferencias radican en una efectiva capacidad de gestión por parte de los municipios a cargo del $\mathrm{PJ}$, mientras que ocurre lo contrario en los demás. Y en cierta medida lo es, para el caso del Municipio de Isla del Cerrito.

Cuadro 10. Programas en Gestión según Partidos Políticos a cargo del Gobierno Municipal. (Fuente Elaboración Propia).

\begin{tabular}{ll}
\hline Partido Político & En Gestión \\
\hline Unión Cívica Radical & 3 \\
Acción Chaqueña & 3 \\
Partido Justicialista & 18 \\
\hline
\end{tabular}

Si los mecanismos para la obtención de estos recursos son claros, ya que se radican en los ministerios del Estado Nacional y los municipios con autonomía institucional pueden contraer obligaciones, podría «aceptarse» que unos municipios son más "capaces" que otros al momento de atraer recursos para sus comunidades. De ahí las desigualdades observadas en los cuadros precedentes. Cabría la posibilidad de suponer que el origen de las desigualdades en la distribución no obedecería al aceitado mecanismo del corporativismo político (poniendo en duda nuestra asunción inicial) sino a la debilidad en la gestión de los municipios menos favorecidos en la asignación de programas. 
¿Debemos atribuir entonces a la mayor capacidad de gestión de que los municipios oficialista prevalezcan sobre los restantes municipios opositores? Cabría también preguntarse ¿Qué evidencias existen de que el corporativismo es la modalidad de la acumulación política que favorece a los municipios oficialista en desmedro de los opositores?

En el Cuadro 11 hemos elegido el artificio de la ponderación para procurar algunas evidencias que den respuestas a estas preguntas. Adoptamos la relación de que por cada US\$20 mil invertidos por la nación, en distintos programas, equivalen a un valor 1 (uno) para hacer más explícita la correlación.

Cuadro 11. Municipios agrupados por Departamentos, según Subsidios recibidos, Signo Partidarios y Ponderación de Inversiones.(Fuente Elaboración Propia).

\begin{tabular}{|c|c|c|c|}
\hline & ATN & $\begin{array}{l}\text { Signo } \\
\text { Partidario }\end{array}$ & $\begin{array}{l}\text { Ponderación 1: } 20.000 \\
\$\end{array}$ \\
\hline \multicolumn{4}{|l|}{ Depto. BERMEJO } \\
\hline La Leonesa & NO & UCR & 12,1 \\
\hline Las Palmas & $S / D$ & $\mathrm{PJ}$ & 17 \\
\hline Isla Del Cerrito & sí & $\mathrm{PJ}$ & 152,5 \\
\hline $\begin{array}{l}\text { Depto. } \\
\text { FERNANDO }\end{array}$ & \multicolumn{3}{|c|}{ SAN } \\
\hline Fontana & sí & $\mathrm{PJ}$ & 5,75 \\
\hline \multicolumn{4}{|l|}{ Depto. LIBERTAD } \\
\hline Colonia Popular & sí & $\mathrm{PJ}$ & 10 \\
\hline Laguna Blanca & NO & $\mathrm{ACHA}$ & 5,2 \\
\hline \multicolumn{4}{|c|}{ Depto. $1^{\circ}$ DE MAYO } \\
\hline Margarita Belén & sí & $\mathrm{PJ}$ & 23 \\
\hline Colonia Benítez & NO & $\mathrm{ACHA}$ & 2,1 \\
\hline
\end{tabular}

Ref.: ATN: Aportes del Tesoro Nacional subsidios; PJ: Partido Justicialista; UCR: Unión Cívica Radical; ACHA: Acción Chaqueña.

La distribución desigual de los recursos para el desarrollo tiene dos fuentes:

n La asignación discriminatoria (como habíamos adelantado en el Cuadro 4 de subsidios bajo la justificación de aliviar situaciones financieras graves de los municipios. No siempre queda demostrado la justificación real de estas asignaciones, ya que en igual o peores condiciones se encuentran otros municipios no oficialistas, pero al ser una atribución discrecional del Poder Ejecutivo Nacional, aparece como una herramienta clara de favoritismo y reproducción política de éste.

n Como habíamos presentado anteriormente, los recursos para el desarrollo tienen una fase equitativa, cuando se los analiza desde la cantidad nominal de programas. Pero al ponderar las asignaciones, las inequidades aparecen más claras y por lo tanto podemos establecer comparaciones y mediciones que fundamentan al menos en ésta instancia las proposiciones que sostiene este trabajo.

La columna de las ponderaciones presenta el atributo de que las inversiones realizadas en los municipios oficialista son marcadamente superiores a los de sus vecinos de su mismo departamento, encontrando picos notables como el caso del Departamento Bermejo, o disparidades importantes como en el Dto. $1^{\circ}$ de Mayo.

La reproducción política del partido gobernante prima al momento de las asignaciones, ya que al ser éstas no reintegrables -salvo un programa en el municipio de Margarita Belén- no guarda relación con los criterios "administrativistas-eficientistas ", por lo tanto sirven a este propósito. 
Mientras que los programas que se dirigen a más largo plazo, que tienden al fortalecimiento económico institucional de los municipios y sus comunidades, no aparecen en nuestros análisis porque simplemente no son considerados como posibilidad objetiva por parte de los municipios por los requerimientos que establece la Nación para su acceso.

La no-promoción o dicho de otra manera, los cambios en los requerimientos del acceso a programas a más largo plazo no es posible en las actuales condiciones de la relación Estado-Municipios, ya que entrarían en contradicción con la modalidad corporativa e inmediatista de reproducción política de este gobierno.

Por lo tanto se prefiere el subsidio directo, por el lapso de tres meses, que se consumen y nuevamente se presenta otra posibilidad de renovación por otro periodo similar, como modalidad de disciplinamiento político aplicado por el gobierno Nacional. Dentro de esta lógica toda perspectiva de inversiones de Bienes de Capital o Programas de Capacitación Múltiples de mediano plazo no tiene sentido, de allí la carencia de éstos.

\section{CONCLUSIONES}

En la primera parte de este trabajo presentamos el patrón de conformación económica de los municipios, en particular los orígenes de sus ingresos, y de sus egresos. Concluimos que los municipios analizados comparten una alta vulnerabilidad en la conformación de los ingresos por una parte y por otra una escasa disponibilidad para nuevos endeudamientos y aumentos en las inversiones, constituyendo el principal escollo estructural a resolver.

Si aceptamos que es una condición compartida por todos los municipios, y que los favoritismos políticos no solucionan esta traba al desarrollo de éstos, se presenta entonces un campo en común para los municipios que va más allá de los límites de la filiación política.

Se trata de establecer un elemento común, convergente para que los fondos estatales sean dirigidos a más largo plazo y que contemplen la reestructuración de sus economías y al diseño de nuevas formas societales del municipio y su comunidad.

Los municipios con nuevos mecanismos societales democráticos deberían abordar el problema de los plazos inmediatos de la distribución de los recursos de los programas, que atienden problemas coyunturales. Hacer evidente de que se esta manipulando recursos sociales y no partidarios, es la prioridad.

Que el problema de las desigualdades pertenece al conjunto social y no esta en manos de un partido el de solucionar esto a través del asistencialismo.

Exigir complementariedad en la distribución de estos recursos. Aquí potencialmente estamos frente a la conformación de nuevos territorios[15] que traspasan las divisiones administrativas de los municipios, potenciando las relaciones sociales que existen en estas pequeñas localidades (mientras que un programa beneficia a un miembro de una familia, perjudica a otro que reside en el municipio vecino al cual no se asiste).

Con estos esquemas tenderemos a separar por un lado la acción propia del Municipio, y por otro él de, la reproducción política del partido a cargo del gobierno municipal, ubicando a éste en su verdadera dimensión, la de influenciar con las ideas respecto de la gestión política -administrativa de la cosa pública.

Los municipios deberán integrar problemáticas y soluciones propias de la escala intermunicipal, sintetizando planteamientos de necesidades y prioridades de la inversión pública en el corto plazo frente a las carencias de estas particularidades que tienen los programas nacionales.

De esta manera se tendería a cambiar la lógica distributiva que obedece más a situaciones de oportunidad de disponibilidad de los recursos más que a prioridades locales. Abundan ejemplos en este sentido (pequeñas terminales de transporte público en localidades de muy baja frecuencia de servicios, subsidios para el tendido de red eléctrica rural, sin demanda real y potencial). 
Mientras que los municipios llevan adelante pequeñas y medianas obras originadas por necesidades locales, cuyos plazos de terminación se extiende al infinito por falta de recursos pudiendo incluir a estas en la integralidad del plan que mencionábamos.

Como comentario final queremos dejar establecido que el presente trabajo representa una pequeña fase intermedia de un proceso investigativo que tiene ambiciones en dos campos: el primero, buscar evidencias de trabajos de teóricos sociales latinoamericanos que nos han servido de referencia, y segundo articular con los municipios implicados en nuestra investigación un espacio de intercambio en el cual ambas partes puedan realimentarse para repensar alternativas propias que nos expliquen y nos den herramientas para navegar contra la corriente cada vez menos hegemónica del liberalismo en nuestros países.

Hemel Hempstead, marzo ‘01

\section{AGRADECIMIENTOS}

En 1995, el Menemismo y las políticas sociales dictadas por los organismos internacionales del crédito estaban en su apogeo y por tanto su hegemonía era indiscutible. Hacer una investigación empírica para conocer la situación de estas a escala municipal pudo ser llevada adelante gracias a dos colegas que por razones distintas consideraron oportuno y razonable encarar este trabajo, estos fueron los arquitectos Víctor Pelli y Eduardo Buttice, a quienes agradezco el apoyo de entonces.

El trabajo se ha enriquecido gracias a las lecturas y comentarios de las siguientes personas, Elena Lucca, Marga Pelli, Gustavo Actis, Leonardo Gómez, Andrea Benítez, quienes quedan totalmente eximidos de los errores que el trabajo pueda tener del cual soy el único responsable.

\section{BIBLIOGRAFÍA}

CORAGGIO, J. L.

1991. “LAS DOS CORRIENTES DE DESCENTRALIZACION EN AMERICA LATINA” en: Ciudades sin Rumbo. Investigación urbana y proyecto popular, SIAP_CIUDAD, Quito.

NICOLÁS, H.D.

1995. "DESEQUILIBRIOS ESTRUCTURALES Y DESIGUALDADES REGIONALES: Alternativas para el Territorio Mexicano", en CALVA, J. L. y AGUILAR G, ed., DESARROLLO REGIONAL Y URBANO. Tendencias y Alternativas. T. 1. Juan Pablo Editor. México.

GARCÍA DELGADO, D. R.

1994. ESTADO \& SOCIEDAD: La Nueva Relación a Partir del Cambio Estructural. Tesis Norma Ed. Bs. As.

RAMIREZ, R.

1997. "LOCAL GOVERNANCE MODEL: DECENTRALISATION AND URBAN POVERTY ERADICATION". Inédito. Development Planning Unit, University College Lon., Febrero.

ROZÉ, J. P,

1992. "CONFLICTOS AGRARIOS EN LA ARGENTINA: EI Proceso Liguista". Centro Editor de América Latina. Bs. As.

ZAPATA, J. A.

1998 "Financiando la Ciudad" en LA CIUDAD EN EL SIGLO XXI: Simposio de Buenas Prácticas en Gestión Urbana en América Latina y el Caribe.Rojas, E. y Daughter. R, editores. Banco Interamericano de Desarrollo, Marzo.

[1] Este trabajo formó parte de la investigación "Del Estado Benefactor al Estado Liberal: El Proceso de Control Territorial en la Provincia del Chaco", Beca Posdoctoral del CONICET 1996-1997. Este artículo fue publicado en la revista Convergencia del Centro de Investigación y Estudios Avanzados de la Facultad de Ciencias Políticas y Administración Pública de la Universidad Autónoma del Estado de México, bajo el título "Efectos del Direccionamiento Desde Arriba Sobre Municipios del Chaco, Argentina 1994-1995". 
[2] En el año 1995, un Secretario de Estado, anuncia un estudio que había sido elevado al Presidente de la Nación, en donde se concluía que a los efectos de reducir gastos se sugería la fusión de tres provincias denominadas no viables económicamente, en una sola administración para ahorrarse los gastos de dos administraciones. Los reclamos de las provincias involucradas en el estudio dieron por tierra la pretensión ministerial. Otras reducciones de gastos fueron sugeridas por el mismo sector político a mediados del año 2000. En esta ocasión, al haber fracasado su propuesta de eliminar provincias, el foco de atención estuvo puesto en los poderes legislativos de las 25 provincias, planteando reducir éstas a cinco legislaturas regionales.

[3] La Ley Orgánica Municipal № 4233 establece las siguientes categorías municipales según el número de habitantes: Los municipios de primera categoría son aquellos que superan los veinte mil habitantes. Los de segunda concentran entre cinco mil y veinte mil habitantes. Los de tercera son centros poblacionales de hasta cinco mil habitantes.

[4] “...El Ajuste Estructural en la República Argentina tuvo su expresión mas clara en la ley de Reforma del estado, esta apunta a la reestructuración de su aparato productivo y administrativo, con la idea de afirmar la supremacía del mercado por sobre el Estado para optimizar la asignación de recursos". (D. R. García Delgado. 1995a: 86)

[5] Sinopsis Estadística de Argentina. Dirección de Difusión del Instituto de Estadísticas y Censo de la República, 1997.

[6] Una importante aportación al tema esta contenido en Ciudades intermedias de América Latina y El Caribe: Propuestas para la gestión urbana. Trabajo compilado por Ricardo Jordan y Daniela Simioni, de la Comisión Económica para América Latina y el Caribe (CEPAL) (1998), financiado por el Ministero degli Affari Estri Cooperazione Italiana.

[7] Seminario Internacional. Programa UIA - CIMES. "EI Rol de las Ciudades Intermedias en Iberoamericana". Resistencia, Chaco, Argentina. Diciembre, 2000.

[8] Acción Chaqueña, liderado por el ex-Coronel Ruiz Palacios, ex Gobernador durante la Dictadura Militar, denunciado en la Comisión Nacional de Desaparecidos. Aliado circunstancial a nivel Nacional con el Partido Justicialista del Presidente Menem.

[9] Nos referimos a las presiones ejercidas desde el Estado Nacional a la Provincia para que esta siga los lineamientos de la primera, en particular, realizar los cambios constitucionales a nivel Provincial que permitieran la privatizaciones de servicios públicos como así también la privatizaciones de los Bancos Provinciales, que en el caso de la Provincia del Chaco no estaban permitidas por su constitución. A nivel Municipal existía el impedimento legal de disponer del patrimonio municipal por parte de sus autoridades, obstáculo que se removió posteriormente, como veremos en los tópicos siguientes.

El fundamentalismo economicista perdió aquí otra oportunidad, alzando banderas de privatización contra empresas provinciales rentables, como las de Agua y Luz Eléctrica. El Banco Provincial corrió otra suerte, altamente deficitario, por malas administraciones, fundamentalmente del período militar, se "privatiza" mediante una ley, vendiendo la mitad del paquete del $51 \%$ que tenia el estado provincial al Gremio de los Trabajadores del Banco.

[10] El entusiasmo se basaba en que por diversas fuentes y niveles, el estado nacional enviaba señales de que los municipios podría acceder a créditos y ayuda internacional (PROVINCIAS I), pero la condiciones para esto era las modificaciones legales descriptas.

[11] Durante este período, la Coparticipación Federal constituyó una herramienta de negociación política por parte del Estado Nacional, con las provincias opositoras, sobre todo cuando el Poder Ejecutivo necesitaba de los votos de los senadores y diputados nacional es para aprobar determinadas leyes que requerían quórum ampliado. Los adelantos o retrasos en el pago de estos fondos a las provincias era el método empleado de disciplinamiento, en abierta violación a las leyes vigentes.

[12] Dos casos son ejemplo de esta afirmación, cuando los municipios de Fontana y Las Palmas, entran en graves problemas institucionales y económicos. La primera por corrupción administrativa y disputas políticas que derivó en intervención por parte del gobierno provincial, y la segunda, por cerrarse una industria que daba puestos de trabajos al $50 \%$ de los habitantes del pueblo, hizo que las autoridades municipales empezaran a incrementar su planta de trabajadores para absorber las presiones sociales derivadas del cierre de la fabrica, que en el plazo inmediato llevó a gravísimos problemas económicos a éstas.

[13] Se han realizado variados intentos de "privatizar" parte de las actividades municipales, el más remanído fue el cobro de impuestos, pasando todo el personal municipal a esa área reconvirtiéndolos en "Sociedades de Responsabilidad Limitada", o "Cooperativas de trabajadores" con fondos provenientes de un programa nacional que subsidiaba el capital inicial de estas nuevas empresas.

[14] Si bien no es el objetivo del presente trabajo desplegar esta relación, tenemos evidencia a través de la encuesta realizada, que los otorgamiento de los subsidios están en relación a fechas electorales, que varían en cantidad y calidad de acuerdo a la importancias de estas, (si son provinciales ó nacionales).

[15] Una interesante mención de esto se encuentra para el caso de México en Nicolás (1995: 31): "En las últimas dos décadas, se ha hablado de la inadecuación de la división municipal de Oaxaca por ejemplo: pero ¿una adecuación para quienes? Con toda seguridad para los que consideran que la planificación y la administración desde arriba exigen un orden racional, dimensiones aceptables y números reconocibles. Para aquellos que viven la realidad de estos municipios, la división municipal es el reflejo de la 
existencia de comunidades realmente existentes, con problemas sin duda diferentes que son el reflejo de la micro-historia de microsociedades, las que finalmente son los agentes reales de la vida de una Nación". 